\title{
Using semi-implicit representation of algebraic surfaces
}

\author{
Laurent Busé \\ INRIA Sophia-Antipolis, GALAAD, \\ 2004 route des Lucioles, B.P. 93, \\ 06902 Sophia-Antipolis, Cedex France. \\ Laurent.Buse@sophia.inria.fr
}

\author{
André Galligo \\ Université de Nice Sophia-Antipolis, \\ Parc Valrose, BP 71, \\ 06108 Nice Cedex 02, France. \\ galligo@math.unice.fr
}

\begin{abstract}
In [3] we introduced a new general representation of algebraic surfaces, that we called semi-implicit, which encapsulates both usual and less known surfaces. Here we specialize this notion in order to apply it in Solid Modeling: we view a surface in $\mathbb{R}^{3}$ as a one-parameter (algebraic) family of algebraic low-degree curves. The paper mainly addresses the topic of performing the usual CAD operations with semi-implicit representation of surfaces. We derive formulae for computing the normal and the curvatures at a regular point. We provide exact algorithms for computing selfintersections of a surface and more generally its singular locus. We also present some surface/surface intersection algorithms relying on generalized resultant calculations.
\end{abstract}

\section{Introduction and examples}

Evolving curves occur in a wide variety of settings and were also used to describe boundaries of volumes. This gave rise in shape description to the natural idea of active contours. This paradigm has received various mathematical interpretation ranging from meshes to level set methods. We aim to develop an algebraic and geometric interpretation of this paradigm in order to contribute with new models depending on a reduced number of parameters having a rich geometry and on which one can perform efficiently the usual CAD (Computer Aided Design) operations.

In the sequel we first give precise definitions and basic properties of ours semi-implicit surfaces and provide an algorithm to compute their implicit representation. Then we mention the approximation and interpolation problems of a point cloud by a semi-implicit surface, illustrating our approach on a piece of the classical example of Stanford's bunny. Finally, we provide formulae for computing the equation of the tangent plane together with the second fundamental form of a semi-implicit surface at a regular point, give an algorithm to compute the self-intersection and singularity locus of a semi-implicit surface, and describe resultant-based methods in order to solve intersection problems.

A semi-implicit representation of a surface consists in representing this surface as a parameterized family of implicit space curves. Actually all algebraic surfaces $S$ in $\mathbb{R}^{3}$ can be seen as such a family of curves, i.e. admits a semiimplicit representation, by cutting it with planes of equations $z=z_{0}$ with $z_{0} \in \mathbb{R}$. Similarly revolution surfaces can easily be seen "semi-implicitly". In classical geometry such a representation already appeared, for instance for conic surfaces [6]. Here is an example showing the rich geometry involved in these representations.

A linear family of conics is a surface obtained as the image of a regular map (without base points)

$$
\begin{aligned}
\mathbb{P}^{1} \times \mathbb{P}^{1} & \stackrel{\phi}{\rightarrow} \mathbb{P}^{3} \\
(s: t) \times(u: v) & \mapsto\left(f_{0}(s, t ; u, v): \cdots: f_{3}(s, t ; u, v)\right),
\end{aligned}
$$

where polynomials $f_{i}(s, t ; u, v)$ are bi-homogeneous of bidegree $(1,2)$. For all fixed $\left(s_{0}: t_{0}\right) \in \mathbb{P}^{1}$ the image of $\phi_{\mid\left(s_{0}: t_{0}\right)}$ is a conic $C_{\left(s_{0}: t_{0}\right)}$ in $\mathbb{P}^{3}$ which is, as all conics in $\mathbb{P}^{3}$, contained in a plane that we denote $H_{\left(s_{0}: t_{0}\right)}$. We thus have a family $H$ of planes parameterized by $\mathbb{P}^{1}$; it corresponds to a bi-homogeneous polynomial $L(x, y, z, w ; s, t)$, linear in $x, y, z, w$ and of degree $\leq 3$ in $s, t$ (this follows immediately from the definition of $\phi$ ). Consequently linear families of conics are contained in a larger class of surfaces which are semi-implicitly represented by a family of planes $H$ and a family of surfaces of degree 2 given by a bi-homogeneous polynomial $C(x, y, z, w ; s, t)$ of bi-degree $(2,2)$. Observe that $C_{\left(s_{0}: t_{0}\right)}=C\left(x, y, z, w ; s_{0}, t_{0}\right) \cap L\left(x, y, z, w ; s_{0}, t_{0}\right)$ (set-theoretically at least). Let us comment how the degree of the family $H$ in variables $s, t$ affects the geometry of the associated surface. We have a map

$$
\theta: \mathbb{P}^{1} \rightarrow \mathbb{P}^{3^{\star}}:(s: t) \mapsto H_{\left(s_{0}: t_{0}\right)}
$$

whose image is a curve $\Gamma$ in $\mathbb{P}^{3^{\star}}$ (where ${ }^{\star}$ stands for the dual), assuming that $L$ does not have an irreducible factor 
independent of $x, y, z, w$. Thus if $\operatorname{deg}(\Gamma)=1$ then we deduce that $H$ has a fixed line, and if $\operatorname{deg}(\Gamma)=2$ then $H$ as a fixed point.

\section{Definition}

An implicit representation of a surface $S$ in $\mathbb{P}^{3}$ consists in viewing it as a closed subvariety of $\mathbb{P}^{3}$, i.e. described as the zero locus of a non-zero homogeneous polynomial in $\mathbb{C}[x, y, z, w]$. A semi-implicit representation basically consists in viewing a surface $S \subset \mathbb{P}^{3}$ as the projection on the first factor of a certain closed subvariety $\mathcal{Z}$ of $\mathbb{P}^{3} \times \mathbb{P}^{1}$.

Definition 2.1 We call a semi-implicit representation of an algebraic surface $S \subset \mathbb{P}^{3}$ a couple of bi-homogeneous polynomials $F(x, y, z, w ; s, t)$ and $G(x, y, z, w ; s, t)$ defining a closed subvariety $\mathcal{Z} \subset \mathbb{P}^{3} \times \mathbb{P}^{1}$ such that its projection on the second factor is surjective and is $S$ on the first factor. If $F$ is linear in the homogeneous variables $x, y, z, w$ then the semi-implicit representation is called linear.

It is possible to give a similar definition of more general semi-implicit representations involving more than two equations (see [3]). Passing from a semi-implicit representation to an implicit representation of $S$ is a useful operation, especially for intersection algorithms. One can complete it as follows.

Proposition 2.2 Let $S$ be a surface semi-implicitly represented by both bi-homogeneous polynomials $F(x, y, z, w ; s, t)$ and $G(x, y, z, w ; s, t)$ of respective bi-degree $\left(k_{1}, d_{1}\right)$ and $\left(k_{2}, d_{2}\right)$, then $S$ is of degree $k_{1} d_{2}+k_{2} d_{1}$ and the Sylvester resultant of $F$ and $G$ with respect to the homogeneous variables $s, t$ gives an implicit representation of $S$.

Proof. This follows from standard properties of the Sylvester resultant. We refer the reader to [5, chapter $3, \S 1]$ for a detailed description of the Sylvester matrix, whose determinant gives the so-called Sylvester resultant. (see e.g. [5]).

Notice that in $\mathbb{R}^{3}$ one obtains all algebraic surfaces but with different degrees. However only rational surfaces may be parameterized (that is only surfaces with zero genus). Thus we can handle more general surfaces with semiimplicit representations than parameterized representations.

\section{Point data approximation}

Point data approximation is an important subject in CAD and has been extensively studied during the last 40 years. One particular class of methods attacks the problem by considering parallel slices of the object and then combining the individual reconstructions of each slice in the third direction. See e.g. $[1,7,8]$ and references therein. In our settings, and dealing more with a representation problem than a reconstruction problem (we assume known some topological informations), this amounts to consider an approximate candidate surface in a linear semi-implicit representation defined by two equations in $\mathbb{R}^{3} \times \mathbb{R}^{1}$. One of an evolving plane $H(x, y, z ; t)$ and one of an evolving surface $C(x, y, z ; t)$ which will delimit a "moving contour" curve in that plane.

The approximation process requires to combine two successive marching algorithms. The first one amounts to define a curve in the dual space of planes in $\mathbb{R}^{3}$ giving a "good ondulation" to the planes $H_{t}$ which will contain the active contours. The second one proceeds by projection on such a $H_{t_{0}}$, for some discretised values of $t \in \mathbb{R}$, of a near-by portion of the 3D-point cloud to give a $2 \mathrm{D}$ point cloud which is later approximated by a portion of an algebraic curve of low degree with a controlled typical shape. For complicated shapes, we do not pretend that this is an easy and convergent process. However, this paradigm leaves open the possibility of a fruitful compromise with a rich geometry between the rigidity (but good understanding) of algebraic geometry and the possibility to decompose a complicated object into a (small) number of well controlled patches.

As an application of this previous interpolation method we were interested in a semi-implicit representation of a rabbit ear given by a scattered data representation which consists of 927 points. This is a toy example which could be easily complicated, but this is not yet our current purpose. Cutting by equally distributed horizontal planes, we formed 20 sets of 45 co-planar points. The approximation of these 45 points in each "slice" by an algebraic curve implicitly represented is the more time-consuming step of the process. A way to perform it is to use a particular family of planar quartic curves, called binoids, whose real part is formed by a multiple point and an oval (the oval modelizes an active contour and the multiple point its skeleton following the general idea of Blum [2]).

This conference sketch paper does not present achieved works and implementations for this difficult problem of point data approximation but a new framework and targets that we plan to develop further and discuss with researchers in the field. Efficiency will be addressed in future works.

\section{Usual differential geometric invariants}

Given a semi-implicitly represented surface, it is possible to compute at any regular point the usual differential geometric invariants such as the equation of the tangent plane, and thus the normal, or the second fundamental form, and thus the curvatures.

Let us do it for a surface $S$ passing by the origin and represented by a family of plane curves parameterized by 
$t$ (the origin being obtained for $t=0$ ). $S$ is given by the two equations: $L(x, y, z, t)$ of degree one in $x, y, z$ and of possibly higher degree in $t ; F(x, y, z, t)$ of any degree in $x, y, z, t$. With our hypothesis they satisfy $L(0,0,0,0)=0$, and $F(0,0,0,0)=0$.

The tangent space of $S$ at the origin is generically the projection of the tangent space at the surface defined by $L$ and $F$ at $(0,0,0,0)$ in $\mathbb{R}^{4}$. So in order to compute the tangent space we can truncate $L$ and $F$ and keep only their affine Taylor expansions, that we call $L_{1}$ and $F_{1}$. To be more specific let

$$
L_{1}:=l x+m y+n z+p t, \quad F_{1}:=a x+b y+c z+d t,
$$

then the equation of the tangent space is:

$$
T:=(-p a+d l) x+(-p b+d m) y+(d n-p c) z .
$$

If $p$ and $d$ are both zero, then $L_{1}$ and $F_{1}$ should be proportional in order that the origin is non singular on $S$, in that case we keep either equation. The three coefficients of $T$ define the coordinates of the normal of $S$ at the origin.

The computation of the second fundamental form is more complicated. It amounts to compute an implicit equation of $S$ near by the origin, truncated at orders greater than three. We take the Taylor expansions of $L$ and $F$ at order 3 in $x, y, z$ (we should not truncate also in $t$ ). Let us call them $L_{2}$ and $F_{2}$. They are two polynomials in $x, y, z, t$. We use a resultant to eliminate $t$ between $L_{2}$ and $F_{2}$ and we get a polynomial $G(x, y, z)$ in $x, y, z$ whose degree depends on the degrees in $t$ of $L_{2}$ and $F_{2}$. Then we compute a Taylor expansion at order 3 of $G$ at the origin and get a polynomial of degree 2 which writes $T+Q_{1}$, with $Q_{1}$ a quadratic form in $x, y, z$. This provides a local equation of $S$ at the origin.

Then it suffices to perform a change of coordinates (which preserves the metric), call $X, Y, Z$ the new coordinates, so that the previous local equation of $S$ at the origin becomes $Z+Q(X, Y, Z)=0$, where $Q$ is a quadratic form. Finally the second fundamental form for $S$ at the origin is simply $Q(X, Y, 0)$.

\section{Singularities and self-intersection points}

An important problem in Computer Aided Geometric Design is the detection of singularities and self-intersection points of a 3D-surface. We describe a method to complete such a detection in case the considered surface is semiimplicitly represented.

Let $S$ be a surface semi-implicitly represented by both polynomials $F(x, y, z, w ; s, t)$ and $G(x, y, z, w ; s, t)$ of respective bi-degree $\left(k_{1}, d_{1}\right)$ and $\left(k_{2}, d_{2}\right)$. A given point $q \in$ $S \subset \mathbb{P}^{3}$ is a self-intersection point if there exist two distinct points $\left(s_{1}: t_{1}\right)$ and $\left(s_{2}: t_{2}\right)$ in $\mathbb{P}^{1}$ such that:

$$
F\left(q ; s_{i}, t_{i}\right)=G\left(q ; s_{i}, t_{i}\right)=0, \text { for } i=1,2 .
$$

By proposition 2.2 we know that an implicit equation of $S$ can be obtained as the determinant of the Sylvester matrix of $F$ and $G$ with respect to the homogeneous variables $s, t$. We denote by $R(x, y, z, w)$ this Sylvester matrix and take a given point $p \in \mathbb{P}^{3}$. If $p$ is not on $S$ then clearly the kernel of $R(p)$ is reduced to 0 since its determinant is non-zero. Now if $p$ is on $S$ then obviously the kernel of $R(p)^{t}$ (where $t$ stands for transpose) is not reduced to zero since it contains a multiple of the vector of monomials $\left(s_{0}, t_{0}\right)^{d_{1}+d_{2}-1}$, where $\left(s_{0}: t_{0}\right) \in \mathbb{P}^{1}$ is such that

$$
F\left(p ; s_{0}, t_{0}\right)=G\left(p ; s_{0}, t_{0}\right)=0
$$

(observe that we can consequently compute $\left(s_{0}: t_{0}\right)$ ). If now $p$ is a self-intersection point of $S$ then the dimension of the kernel of $R(p)^{t}$ is at least 2 since this kernel contains both non collinear vector of monomials $\left(s_{1}, t_{1}\right)^{d_{1}+d_{2}-1}$ and $\left(s_{2}, t_{2}\right)^{d_{1}+d_{2}-1}$. Thus a necessary condition for a point $p$ to be a self-intersection point is that $\operatorname{rank}(R(p)) \leq d_{1}+d_{2}-3$. Similarly, if $\left(p ; s_{0}, t_{0}\right)$ is a singular point of the semiimplicit representation such that $F, G, \partial_{s} F, \partial_{s} G$ all vanish at this point then both non-collinear monomial vectors $\left(s_{0}, t_{0}\right)^{d_{1}+d_{2}-1}$ and $\partial_{s}\left(\left(s_{0}, t_{0}\right)^{d_{1}+d_{2}-1}\right)$ are in the kernel of $R(p)$. In other words, singularities and self-intersection points of the semi-implicit representation of $S$ are located on the zero locus of the $\left(d_{1}+d_{2}-2\right) \times\left(d_{1}+d_{2}-2\right)$ minors of the Sylvester matrix $R(x, y, z, w)$ in $\mathbb{P}^{3}$.

\section{Intersecting a semi-implicit surface}

We now investigate the intersection problems between different curves and surfaces. Our aim is to show that semi-implicit representations are well adapted to these operations. We illustrate it on the three main configurations, say the intersection between a semi-implicit surface and a parameterized curve, a parameterized surface and a semi-implicit surface. Hereafter $S$ denotes a surface semi-implicitly represented by both polynomials $F(x, y, z, w ; s, t)$ and $G(x, y, z, w ; s, t)$ of respective bi-degree $\left(k_{1}, d_{1}\right)$ and $\left(k_{2}, d_{2}\right)$.

\subsection{With a parameterized space curve}

Let $g_{0}, g_{1}, g_{2}, g_{3}$, be four homogeneous polynomials in both variables $s, t$ of the same degree $d$, and let $C$ be the parameterized curve (we write here, for simplicity, the affine version of this parameterization, i.e. set $t=1$ and $w=1$ )

$$
C: \quad\left(x=\frac{g_{1}(s)}{g_{0}(s)}, y=\frac{g_{2}(s)}{g_{0}(s)}, z=\frac{g_{3}(s)}{g_{0}(s)}\right) .
$$

We assume w.l.o.g. that there is no base point, i.e. that $\operatorname{gcd}\left(g_{0}, g_{1}, g_{2}, g_{3}\right)$ is a constant. By proposition 2.2 we know that there exists a resultant matrix $R(x, y, z)$ whose 
determinant is an implicit representation of $S$. Now substituting respectively $x, y, z$ by $\frac{g_{1}(s)}{g_{0}(s)}, \frac{g_{2}(s)}{g_{0}(s)}$ and $\frac{g_{3}(s)}{g_{0}(s)}$ we obtain a matrix $R(s)$ depending on the alone variable $s$ that we can decompose as $\mathrm{R}(s)=\mathrm{R}_{d} s^{d}+\cdots+\mathrm{R}_{0}$, where the coefficients $\mathrm{R}_{i}$ are numerical matrices of the same size than $\mathrm{R}(s)$. And we are looking for the values of $s$ such that this surface and the curve intersect at $C(s)$, that is such that the determinant of $\mathrm{R}(s)$ vanishes. This is related to known methods for solving such "equation", we refer e.g. to [4].

\subsection{With a parameterized surface}

Let $f_{0}, f_{1}, f_{2}, f_{3}$ be four homogeneous polynomials of the same degree $d$ in the homogeneous variables $t_{0}, t_{1}, t_{2}$. They define a parameterized surface in $\mathbb{P}^{3}$ (here again we present the affine point of view, setting $t_{0}=1$ and $w=1$ ):

$$
S^{\prime}: \quad\left(x=\frac{f_{1}\left(t_{1}, t_{2}\right)}{f_{0}\left(t_{1}, t_{2}\right)}, y=\frac{f_{2}\left(t_{1}, t_{2}\right)}{f_{0}\left(t_{1}, t_{2}\right)}, z=\frac{f_{3}\left(t_{1}, t_{2}\right)}{f_{0}\left(t_{1}, t_{2}\right)}\right) .
$$

Our goal is here again to represent the intersection curve $\mathcal{C}$ of $S$ and $S^{\prime}$. We assume for simplicity that the parameterization of $S^{\prime}$ is without base point. By Bezout's theorem we deduce that $\mathcal{C}$ is of degree $d^{2}\left(k_{1} d_{2}+k_{2} d_{1}\right)$. By proposition 2.2 we know that there exists a resultant matrix $\mathrm{R}(x, y, z)$ whose determinant is an implicit representation of $S$. Substituting respectively $x, y, z$ by $\frac{f_{1}\left(t_{1}, t_{2}\right)}{f_{0}\left(t_{1}, t_{2}\right)}, \frac{f_{2}\left(t_{1}, t_{2}\right)}{f_{0}\left(t_{1}, t_{2}\right)}$ and $\frac{f_{3}\left(t_{1}, t_{2}\right)}{f_{0}\left(t_{1}, t_{2}\right)}$ we obtain a matrix $\mathrm{R}\left(t_{1}, t_{2}\right)$ depending only on both variables $t_{1}$ and $t_{2}$. Its determinant defines a curve (implicitly represented) which is of degree $d\left(k_{1} d_{2}+k_{2} d_{1}\right)$, that is to say of lower degree than $\mathcal{C}$. This curve is a representation of the intersection curve $\mathcal{C}$ since every point $t_{1}, t_{2}$ such that $\mathrm{R}\left(t_{1}, t_{2}\right)=0$ can be sent on $\mathcal{C}$ by the parameterization of $S^{\prime}$.

This method consisting of representing an intersection space curve by a birational plane curve is very useful in practice. It has been widely studied (see e.g. [4] and references therein) in the context of the intersection of two parameterized surfaces. We thus show here that we can also represent in this process one of the surface semi-implicitly instead of parametrically.

\subsection{With a semi-implicit surface}

In the case of the intersection of two semi-implicit surfaces we can, as in the previous paragraph, obtain a plane curve which is birational to the intersection curve. As previously, to do this we are going to use a resultant. However we need a more general resultant than the Sylvester one: we need a so-called Macaulay resultant for four homogeneous polynomials in four homogeneous variables $[5$, chapter 3 ,
$\S 2]$. If $S^{\prime}$ is a semi-implicit surface defined by both polynomials $F^{\prime}\left(x, y, z, w ; s^{\prime}, t^{\prime}\right)$ and $G^{\prime}\left(x, y, z, w ; s^{\prime}, t^{\prime}\right)$ of respective bi-degree $\left(k_{1}^{\prime}, d_{1}^{\prime}\right)$ and $\left(k_{2}^{\prime}, d_{2}^{\prime}\right)$, it appears that the resultant of $F, G, F^{\prime}$ and $G^{\prime}$ with respect to the homogeneous variables $x, y, z, w$ is a polynomial in $s$ and $s^{\prime}$. It vanishes at a given point $s_{0}, s_{0}^{\prime}$ if and only if both surfaces $S$ and $S^{\prime}$ intersect with these parameters, i.e. there exists (at least) a point $\mathrm{x} \in \mathbb{P}^{3}$ such that

$$
F\left(\mathbf{x} ; s_{0}\right)=G\left(\mathbf{x} ; s_{0}\right)=F^{\prime}\left(\mathbf{x} ; s_{0}^{\prime}\right)=G^{\prime}\left(\mathbf{x} ; s_{0}^{\prime}\right) .
$$

Let us denote by $R\left(s, s^{\prime}\right)$ this polynomial. It defines a plane curve in the plane of coordinates $\left(s, s^{\prime}\right)$ which is in correspondence with the intersection (space) curve of $S$ and $S^{\prime}$. We can therefore, as in the previous paragraph, apply all the techniques developed by many authors on such a representation of the intersection curve.

\section{Conclusion}

In this paper we presented new algebraic models for representing shapes together with efficient algorithms to compute their local differential geometric invariants, their singularity locus and their intersections. To represent a complex shape in our context we will decompose it into smaller parts. Each of these parts should be sliced in order to get a family of curves having similar shapes. There are at least two main next tasks : one is a detection/recovery problem, and the other one is to reconstruct a model from a set of such parts.

\section{References}

[1] C. L. Bajaj, E. J. Coyle, and K. N. Lin. Arbitrary topology shape reconstruction from planar cross sections. Graphical Models and Image Processing, 58:524-543, 1996.

[2] H. Blum. A transformation for extracting new descriptors of shapes. In Models for the Perception of Speech and Visual Form, volume MIT Press of Cambridge, pages 362-380, 1967.

[3] L. Busé and A. Galligo. Semi-implicit representations of surfaces in $\mathbb{P}^{3}$, resultants and applications. To appear in $\mathbf{J}$. of Symbolic Computation.

[4] J. F. Canny and D. Manocha. A new approach for surface intersection. International Journal of Computational Geometry and Applications, 1(4):491-516, 1991.

[5] D. Cox, J. Little, and D. O'Shea. Using algebraic geometry. Graduate Texts in Mathematics. Springer, 1998.

[6] W. Degen. Surfaces with a conjugate net of conics in projective space. Tensor (N.S.), 39:167-172, 1982.

[7] C. Gitlin, J. O'Rourke, and V. Subramannian. On reconstructing polyhedra from parallel slices. International Journal of Computational Geometry and Applications, 6:103-122, 1996.

[8] G. Turk and J. F. O'Brien. Shape transformation using variational implicit functions. In Siggraph' 99 Conference Proceedings, pages 335-342, 1999. 